\title{
Comparative genomic analysis of NAC transcriptional factors to dissect the regulatory mechanisms for cell wall biosynthesis
}

Dongxia Yao ${ }^{1 \dagger}$, Qiang $\mathrm{Wei}^{1+}$, Wenying Xu', Ryan D Syrenne ${ }^{2}$, Joshua S Yuan ${ }^{2}$, Zhen Su ${ }^{1 *}$

From Proceedings of the Ninth Annual MCBIOS Conference. Dealing with the Omics Data Deluge Oxford, MS, USA. 17-18 February 2012

\begin{abstract}
Background: NAC domain transcription factors are important transcriptional regulators involved in plant growth, development and stress responses. Recent studies have revealed several classes of NAC transcriptional factors crucial for controlling secondary cell wall biosynthesis. These transcriptional factors mainly include three classes, SND, NST and VND. Despite progress, most current analysis is carried out in the model plant Arabidopsis. Moreover, many downstream genes regulated by these transcriptional factors are still not clear.

Methods: In order to identify the key homologue genes across species and discover the network controlling cell wall biosynthesis, we carried out comparative genome analysis of NST, VND and SND genes across 19 higher plant species along with computational modelling of genes regulated or co-regulated with these transcriptional factors.

Results: The comparative genome analysis revealed that evolutionarily the secondary-wall-associated NAC domain transcription factors first appeared in Selaginella moellendorffii. In fact, among the three groups, only VND genes appeared in S. moellendorffii, which is evolutionarily earlier than the other two groups. The Arabidopsis and rice gene expression analysis showed specific patterns of the secondary cell wall-associated NAC genes (SND, NST and VND). Most of them were preferentially expressed in the stem, especially the second internodes. Furthermore, comprehensive co-regulatory network analysis revealed that the SND and MYB genes were co-regulated, which indicated the coordinative function of these transcriptional factors in modulating cell wall biosynthesis. In addition, the co-regulatory network analysis revealed many novel genes and pathways that could be involved in cell wall biosynthesis and its regulation. The gene ontology analysis also indicated that processes like carbohydrate synthesis, transport and stress response, are coordinately regulated toward cell wall biosynthesis.
\end{abstract}

Conclusions: Overall, we provided a new insight into the evolution and the gene regulatory network of a subgroup of the NAC gene family controlling cell wall composition through bioinformatics data mining and bench validation. Our work might benefit to elucidate the possible molecular mechanism underlying the regulation network of secondary cell wall biosynthesis.

\footnotetext{
* Correspondence: zhensu@cau.edu.cn

† Contributed equally

'State Key Laboratory of Plant Physiology and Biochemistry, College of Biological Sciences, China Agricultural University, Beijing 100193, China Full list of author information is available at the end of the article
} 


\section{Background}

As a potential replacement for traditional fossil fuels, biofuels have received increased public and scientific attention in recent years [1]. The current first generation biofuel is based on sugar and starch derived from feedstocks such as sugrarcane and corn; however, this platform is not sustainable for various reasons. Lignocellulosic biomass has been proposed as the major feedstock for second generation biofuels to enable the transition from fossil fuel-based energy to renewable energy for the various economic and environmental advantages gained over first generation biofuels $[1,2]$. Generally speaking, lignocellulosic biomass is composed of cellulose, hemicellulose, pectin and/or lignin, but the amount and ratios between the components can vary considerably [3]. In addition to the aforementioned components, even the amorphous portions of cellulose are purportedly important for lignocellulosic conversion to biofuel [4-7]. Since secondary cell walls in fibres and tracheary elements constitute the most abundant biomass produced by plants, it is necessary to elucidate the possible molecular mechanisms underlying the regulation of secondary cell wall biosynthesis for improved plant biomass production.

Plant NAC (NAM, ATAF1/2 and CUC2) domain proteins are one of the largest groups of plant-specific transcriptional factors and are known to play diverse roles in various plant development processes and stress response. NAM (no apical meristem) was the first characterized NAC gene in petunia. The NAM gene product is required for apical meristem formation and correct positioning of the cotyledons during petunia embryogenesis [8]. ATAF1 and ATAF2 are the two NAC genes in Arabidopsis playing negative roles in response to drought and pathogen infection respectively $[9,10]$. CUC2 (CUPSHAPED COTYLEDON 2) gene was also characterized as a NAC gene in Arabidopsis [11]. Arabidopsis RD26 (RESPONSIVE TO DEHYDRATION 26) encodes a NAC domain protein [12] with function in ABA-mediating gene expression under stress conditions [13]. StNAC, one potato NAC gene, was shown to be rapidly and strongly induced by wounding [14]. Over-expression of OsNAC6/SNAC2 in rice can enhance the seedling plants tolerance to drought, salt, and cold stresses $[15,16]$. Recently, accumulating evidence has indicated that a considerable portion of NAC domain proteins play crucial roles in the processes of xylogenesis, fibre development and wood formation in vascular plants [17]. In the model plant Arabidopsis, NST1 (NAC Secondary Wall Thickening Promoting Factor1), NST2 and NST3/SND1 (Secondary Wall-associated NAC Domain Protein1) are key switches in regulating secondary cell wall biosynthesis in a partially redundant manner [18-25]. NST1 and NST2 function redundantly in regulating secondary cell wall thickening in the endothecium of anthers whereas
NST1 and NST3/SND1 were shown to regulate secondary wall thickening in fibres. In Medicago sativa, MtNST1 (Medicago truncatula NAC Secondary Wall Thickening Promoting Factor 1) has been identified as the only homologue of AtNSTs [26]. Loss of function of the single MtNST1 gene resulted in lack of lignifications in interfascicular fibres, loss of anther dehiscence and stomatal phenotypes associated with loss of ferulic acid in guard cell walls. VND6 (Vascular-related NACdomain6) and VND7 are key regulators in protoxylem and metaxylem development. VND6 is specifically expressed in the metaxylem of Arabidopsis primary roots whereas VND7 is expressed in the protoxylem. Recently, the VND6 gene was discovered to regulate xylem formation by directly targeting some genes related to secondary cell wall formation. VND6 also acts as a direct regulator of genes related to programmed cell death [27]. SND2 and SND3 were also found to function in the formation of secondary cell walls in fibres, and were down-stream of NST1 and NST3/VND1. Six NAC genes associated with wood formation in Populus were also reported [28]. Among the six genes, WND2B (Wood-Associated NAC Domain Transcription Factors) and WND6B were functional orthologues of Arabidopsis SND1 and master switches activating secondary wall biosynthesis during wood formation in Populus. Recently, XND1(Xylem NAC Domain1) was reported to influence the differentiation of tracheary elements and xylem development in Arabidopsis by negatively regulating terminal secondary wall biosynthesis and programmed cell death in xylem vessel cells [29].

Although several key switches in regulation of secondary wall formation have been found in the model plants Arabidopsis and Populus, key regulators in other plants and many downstream genes regulated by these transcriptional factors are still not clear. In order to identify key homologue genes and discover the network controlling cell wall biosynthesis, we carried out comparative genome analysis of NST, VND and SND genes across 19 higher plant species. The analysis revealed that the NAC domain transcription factors associated with the secondary cell wall evolutionarily first appeared in Selaginella moellendorffii. In fact, among the three groups, only VND genes were identified in S. moellendorffii, which is evolutionarily earlier than the other two groups. Gene expression analysis was carried out to analyse the regulation of NAC genes associated with secondary cell wall biosynthesis in different tissues and revealed that several of these transcriptional factors were co-regulated. To further characterize the candidate genes involved in the regulation of secondary cell wall biosynthesis, we performed a comprehensive co-regulatory network analysis and discovered that some secondary wall-associated NAC genes and MYB genes were co-regulated. In 
addition, co-regulatory network analysis also revealed many novel genes and pathways that may be involved in cell wall biosynthesis and regulation.

\section{Methods}

Sequence retrieval and phylogenetic analysis

Protein sequences and DNA binding domain alignment of the NAC transcriptional factor gene family were downloaded from Plant Transcriptional Factor Database http://planttfdb.cbi.pku.edu.cn. Multiple alignments were performed using ClustalX (1.83) software, and the Neighbour-Joining (NJ) method was used to construct a phylogenetic tree. Genes sharing the same clade with the NAC genes controlling cell wall composition from Arabidopsis were chosen for further study, which resulted in 199 proteins across 19 species.

\section{Microarray data analysis}

The expression profiling data was acquired from local and publically available databases (e.g. GEO and AtGenExpress). The signal intensity for each probe set of each GeneChip was extracted by Affymetrix software GCOS (MAS 5.0).

Eisen's cluster software http://rana.lbl.gov/EisenSoftware.htm was applied for cluster analysis. The signal intensities of microarray experiments were directly used for hierarchical clustering analysis. We employed standard data adjustment and SOM (Self-Organizing Map) clustering in precedence of hierarchical clustering to achieve a better grouping result.

Gene ontology (GO) analysis was performed for differentially expressed genes using the EasyGO web server [30]. During GO processing, the statistical test method used was the chi-square test with FDR p-value $\leq 0.05$ as the cut-off.

The gene network data was constructed using Pathway Studio http://www.ariadnegenomics.com/products/pathway-studio/, ATTED http://atted.jp/ and Hans J. Bohnert's paper [31], and the map was constructed using Pathway Studio (version 6.2).

\section{Plant materials}

Seven tissue samples of rice (Oryza sativa subsp. japonica var. Nipponbare) were selected for real-time RTPCR (reverse transcription polymerase chain reaction) analysis. Rice calli were cultured in N6 solid medium [32] and harvested after one month of induction. Root samples were harvested from rice seedlings that were cultured in a growth container for two weeks. The other five samples (penultimate leaf, flag leaf, spikelet, seed and stem) were harvested from rice plant grown for about four months under natural conditions in Beijing, China.

\section{RNA isolation and real-time RT-PCR}

All rice tissue samples were homogenized in liquid nitrogen before isolation of RNA. Total RNA was isolated using TRIZOL reagent (Invitrogen, CA, USA) and purified using Qiagen RNeasy columns (Qiagen, Hilden, Germany). Reverse transcription was performed using Moloney murine leukemia virus (M-MLV; Invitrogen). The cDNA samples were diluted to $8 \mathrm{ng} / \mu \mathrm{L}$ for realtime RT-PCR analysis.

For real-time RT-PCR, triplicate assays were performed on $1 \mu \mathrm{L}$ of each cDNA dilution using the SYBR Green Master Mix (Applied Biosystems, USA, PN 4309155) with an ABI 7900 sequence detection system according to the manufacture's protocol (Applied Biosystems). The gene-specific primers were designed by using PRIMER3 http://frodo.wi.mit.edu/primer3/input. htm. The amplification of $18 \mathrm{~S}$ rRNA was used as an internal control to normalize all data (forward primer, 5'-CGGCTACCACATCCAAGGAA-3' and reverse primer, 5'- TGTCACTACCTCCCCGTGTCA-3'). Genespecific primers are listed in Supplemental Table 1 in additional file 1 . The relative quantification method $(\Delta \Delta C T)$ [33] was used to evaluate quantitative variation between replicates examined.

\section{Results and discussion}

Identification of genes of NAC transcriptional factors controlling the cell wall composition across different species

Plant NAC domain proteins are one of the largest group of plant-specific transcription factors. This study mainly focuses on SND, NST and VNDs. Phylogeny analysis was used to identify the SND, NST and VND members in different species. The NAM domains of all NAC proteins were extracted from the Plant Transcriptional Factor Database http://planttfdb.cbi.pku.edu.cn. Multiple alignments and phylogenetic tree analysis showed that the genes shared the same clades with Arabidopsis SND, NST and VND proteins and thus were used for further analysis. This yielded a total of 199 NAC proteins across 19 species that possibly regulate secondary cell wall biosynthesis. The numbers of potential SND, NST and VND proteins with the evolutionary relationship of different species are summarized in Figure 1, while the number of genes in different species may be limited by the Plant Transcriptional Factor Database or sequencing information. For example, MtNST1 was not included in the Plant Transcriptional Factor Database and so was omitted in this study.

The NAC gene family associated with the secondary cell wall biosynthesis evolutionarily first appeared in $S$. moellendorffii (Figure 1). Among the three groups, only VND proteins appeared in S. moellendorffii, which was evolutionarily earlier than the other two groups. 
Table 1 Gene Ontology enrichment analysis for the gene list in SNDs-related network

\begin{tabular}{|c|c|c|c|c|c|}
\hline $\begin{array}{l}\text { GO acc } \\
\text { num }\end{array}$ & $\begin{array}{l}\text { GO } \\
\text { type }\end{array}$ & GO term & $\begin{array}{c}\text { Query } \\
\text { item }\end{array}$ & $\begin{array}{c}\mathrm{Bg} \\
\text { item }\end{array}$ & FDR \\
\hline GO:0009834 & $P$ & secondary cell wall biogenesis & 12 & 18 & $\begin{array}{c}2.40 \mathrm{E}- \\
22\end{array}$ \\
\hline GO:0009832 & $P$ & plant-type cell wall biogenesis & 13 & 52 & $\begin{array}{c}8.00 \mathrm{E}- \\
18\end{array}$ \\
\hline GO:0045449 & $P$ & regulation of transcription & 17 & 231 & $\begin{array}{c}1.70 \mathrm{E}- \\
14\end{array}$ \\
\hline GO:0007275 & $P$ & multicellular organismal development & 18 & 507 & $\begin{array}{c}3.40 \mathrm{E}- \\
10\end{array}$ \\
\hline GO:0048856 & $P$ & anatomical structure development & 13 & 330 & $\begin{array}{c}3.50 \mathrm{E}- \\
08\end{array}$ \\
\hline GO:0044036 & $P$ & cell wall macromolecule metabolic process & 6 & 40 & $\begin{array}{c}1.90 \mathrm{E}- \\
07\end{array}$ \\
\hline GO:0033692 & $P$ & cellular polysaccharide biosynthetic process & 7 & 77 & $\begin{array}{c}4.30 \mathrm{E}- \\
07\end{array}$ \\
\hline GO:0009699 & $P$ & phenylpropanoid biosynthetic process & 5 & 33 & $\begin{array}{c}2.50 \mathrm{E}- \\
06\end{array}$ \\
\hline GO:0010033 & $P$ & response to organic substance & 9 & 210 & $\begin{array}{c}3.40 \mathrm{E}- \\
06\end{array}$ \\
\hline GO:0006355 & $P$ & regulation of transcription, DNA-dependent & 14 & 770 & $\begin{array}{c}7.20 \mathrm{E}- \\
05\end{array}$ \\
\hline GO:0006950 & $P$ & response to stress & 8 & 456 & $\begin{array}{c}5.60 \mathrm{E}- \\
03\end{array}$ \\
\hline GO:0048513 & $P$ & organ development & 9 & 639 & $\begin{array}{c}1.20 \mathrm{E}- \\
02\end{array}$ \\
\hline GO:0003006 & $P$ & reproductive developmental process & 9 & 740 & $\begin{array}{c}3.10 \mathrm{E}- \\
02\end{array}$ \\
\hline GO:0006810 & $P$ & transport & 7 & 540 & $\begin{array}{c}5.00 \mathrm{E}- \\
02\end{array}$ \\
\hline GO:0003700 & $\mathrm{F}$ & transcription factor activity & 27 & 46 & $\begin{array}{c}3.00 \mathrm{E}- \\
49\end{array}$ \\
\hline GO:0016563 & $\mathrm{F}$ & transcription activator activity & 14 & 24 & $\begin{array}{c}1.10 \mathrm{E}- \\
25\end{array}$ \\
\hline GO:0008471 & $\mathrm{F}$ & laccase activity & 6 & 17 & $\begin{array}{c}3.00 \mathrm{E}- \\
09\end{array}$ \\
\hline GO:0016682 & $\mathrm{F}$ & $\begin{array}{l}\text { oxidoreductase activity, acting on diphenols and related substances as donors, oxygen as } \\
\text { acceptor }\end{array}$ & 6 & 28 & $\begin{array}{c}6.70 \mathrm{E}-\mathrm{-} \\
08\end{array}$ \\
\hline GO:0016679 & $\mathrm{F}$ & oxidoreductase activity, acting on diphenols and related substances as donors & 6 & 37 & $\begin{array}{l}2.70 \mathrm{E}-\mathrm{n} \\
07\end{array}$ \\
\hline GO:0046914 & $\mathrm{F}$ & transition metal ion binding & 8 & 195 & $\begin{array}{c}3.50 \mathrm{E}-\mathrm{n} \\
05\end{array}$ \\
\hline GO:0016757 & $\mathrm{F}$ & transferase activity, transferring glycosyl groups & 11 & 465 & $\begin{array}{c}8.90 \mathrm{E}-\mathrm{n} \\
05\end{array}$ \\
\hline GO:0016491 & $\mathrm{F}$ & oxidoreductase activity & 11 & 555 & $\begin{array}{c}4.00 \mathrm{E}-\mathrm{n} \\
04\end{array}$ \\
\hline GO:0043169 & $\mathrm{F}$ & cation binding & 8 & 306 & $\begin{array}{c}5.30 \mathrm{E}- \\
04\end{array}$ \\
\hline GO:0004553 & $\mathrm{F}$ & hydrolase activity, hydrolyzing O-glycosyl compounds & 8 & 397 & $\begin{array}{c}2.90 \mathrm{E}-\mathrm{n} \\
03\end{array}$ \\
\hline GO:0009055 & $\mathrm{F}$ & electron carrier activity & 5 & 264 & $\begin{array}{c}4.10 \mathrm{E}- \\
02\end{array}$ \\
\hline GO:0005634 & C & nucleus & 21 & 212 & $\begin{array}{c}6.00 \mathrm{E}-\mathrm{-} \\
20\end{array}$ \\
\hline GO:0044425 & C & membrane part & 8 & 364 & $\begin{array}{c}3.30 \mathrm{E}-\mathrm{-} \\
03\end{array}$ \\
\hline Total & & & 131 & 31819 & \\
\hline
\end{tabular}

P: biological process; F: molecular function; C: cellular component; Bg: background 


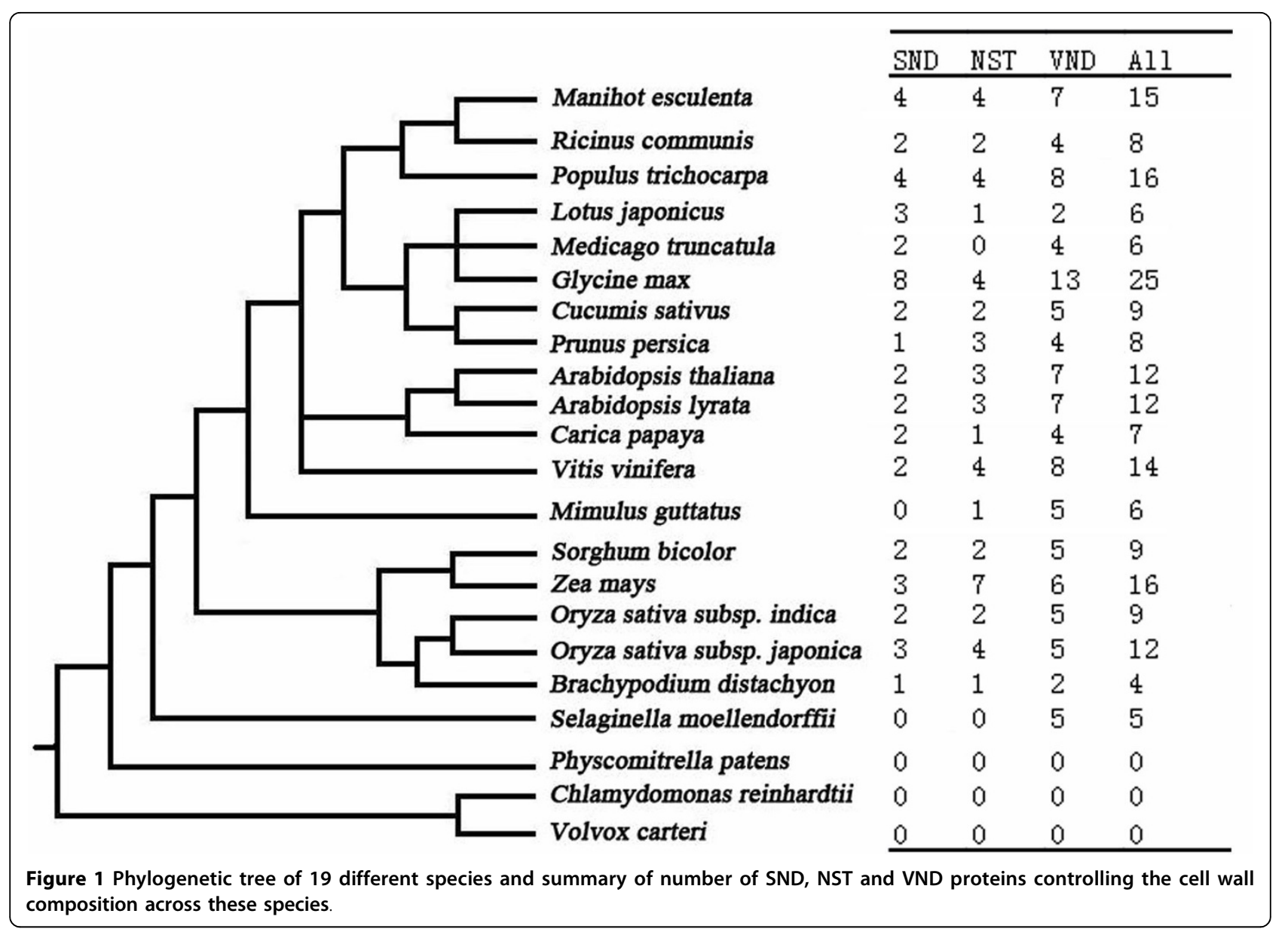

Evolutionary relatedness of SND, NST, VND genes in different species

A phylogenetic analysis of the 199 NAC proteins was performed with ClustalX (1.83) using multiple alignment results. A radial NJ tree was generated (Figure 2), and the phylogeny tree was divided into three classes differentiated by specific conserved domains and highlighted with three different colours. The three subgroups (described in Figure 2) were named NST, SND and VND. The orthologues of NSTs (NST1, NST2 and NST3) included 48 proteins from 17 species. Through phylogeny analysis, NST3 (also named as SND1) orthologues were evolutionally closed to NST subfamily. The SND gene subfamily encompasses 45 proteins from 17 species. This subfamily can also be divided into two subunits: the SND2 and SND3 homologues. In the SND3 homologue subunit, all genes were from dicots; whereas the SND2 homologues were from both dicots and monocots. Compared to SND2 and SND3, the VND subfamily was much larger and included four subunits. The first subunit included homologues of VND7, which were all from dicots. Remarkably, the second subunit only contained five proteins from $S$. moellendorffii. In the other two subunits, VND orthologues demonstrated an interspersed distribution from different species.

\section{Expression patterns of SND, NST and VND genes in Arabidopsis}

To better understand the functions of SND, NST and VND genes, we analysed the gene expression profiles of the 12 NAC genes from Arabidopsis using microarray data, which included eight diverse Arabidopsis tissues with triplicate samples using the Arabidopsis ATH1 array [34]. Hierarchical cluster analysis was conducted and genes with similar expression patterns were clustered together. The five NST and SND genes showed a highly similar expression pattern in the tissue specific experiments (Figure 3). They were preferentially expressed in stems, especially the second internodes, and were relatively less expressed in leaves, flowers, roots, hypocotyls and mature pollens. In siliques, these genes were expressed at an average level. In contrast, the VND genes exhibited very different expression profiles in different tissues, except for VND6 and $V N D 7$ which were preferentially expressed in the stem. For example, VND1 showed expression in flowers, roots, hypocotyls and first nodes of the stem while VND3 and VND4 


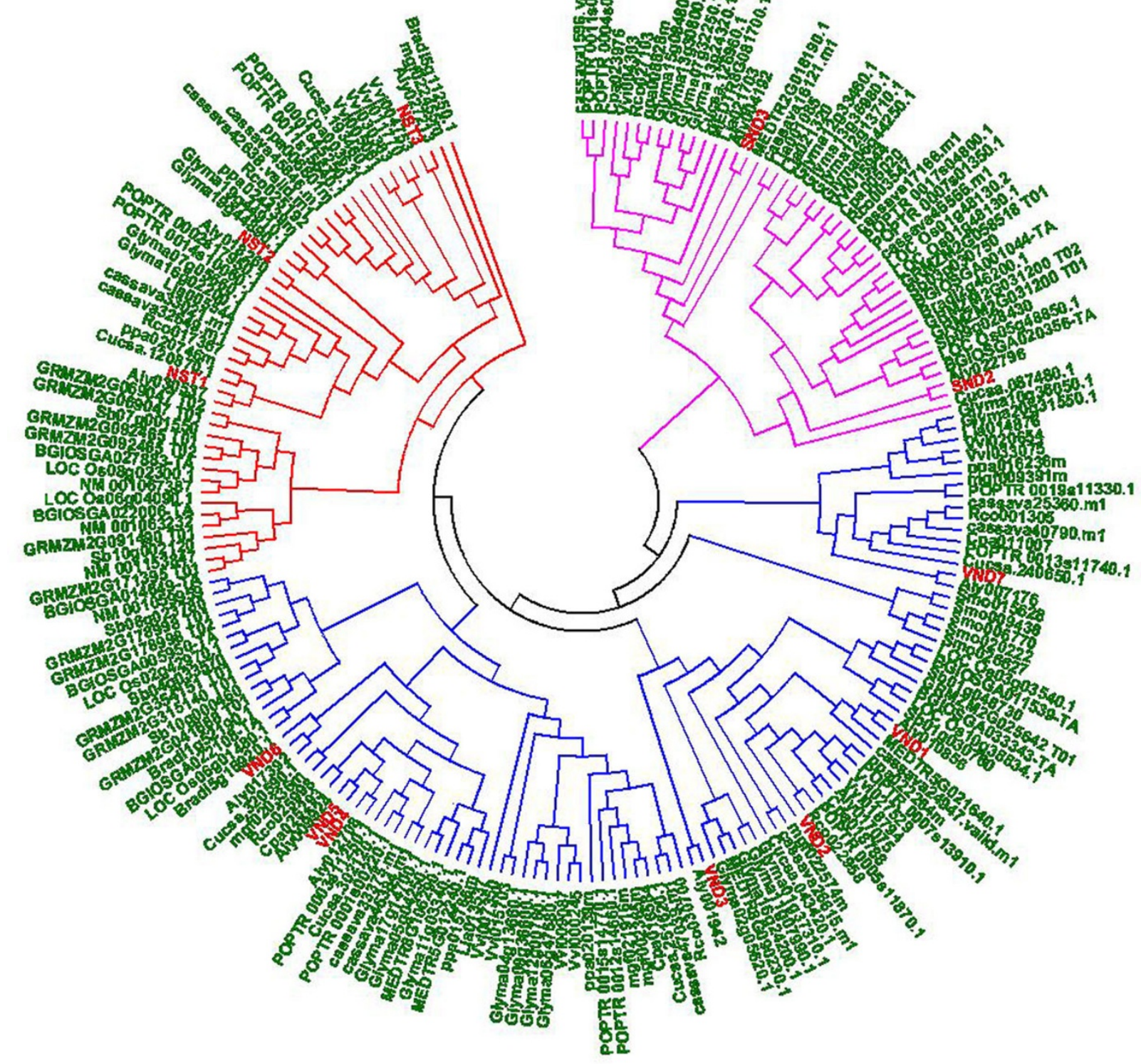

Figure 2 Phylogenetic tree of SND, NST and VND proteins across the 19 different species. The three clades are indicated in specific colours. Members of SND, NST, and VND protein from Arabidopsis are denoted in red. The detailed information of these proteins was listed in Supplemental Table 3 in additional file 3.

were mainly expressed in mature pollens, and second internodes of the stem. In general, all seven genes were expressed in hypocotyls. With the exception of $V N D 3$ and $V N D 4$, the other VND genes were highly expressed in roots. VND2 was also highly expressed in mature pollen together with $V N D 3$ and $V N D 4$. Although the three classes of NAC genes showed different expression profiles, they all showed lower expression levels in leaves. These microarray results suggest that VND genes may have diverse functions in different developmental stages or cellular processes. The cluster analysis also revealed that several of these transcriptional factors were co-regulated.

\section{Expression patterns of SND, NST and VND orthologue genes in rice}

Rice is a model monocot plant for molecular and genetic studies. Recently, large sets of rice tissue-specific microarray data have been available in the GEO database. GSE19024 [35] was selected, including 39 tissues covering the entire tissue culture process and life cycle, from 


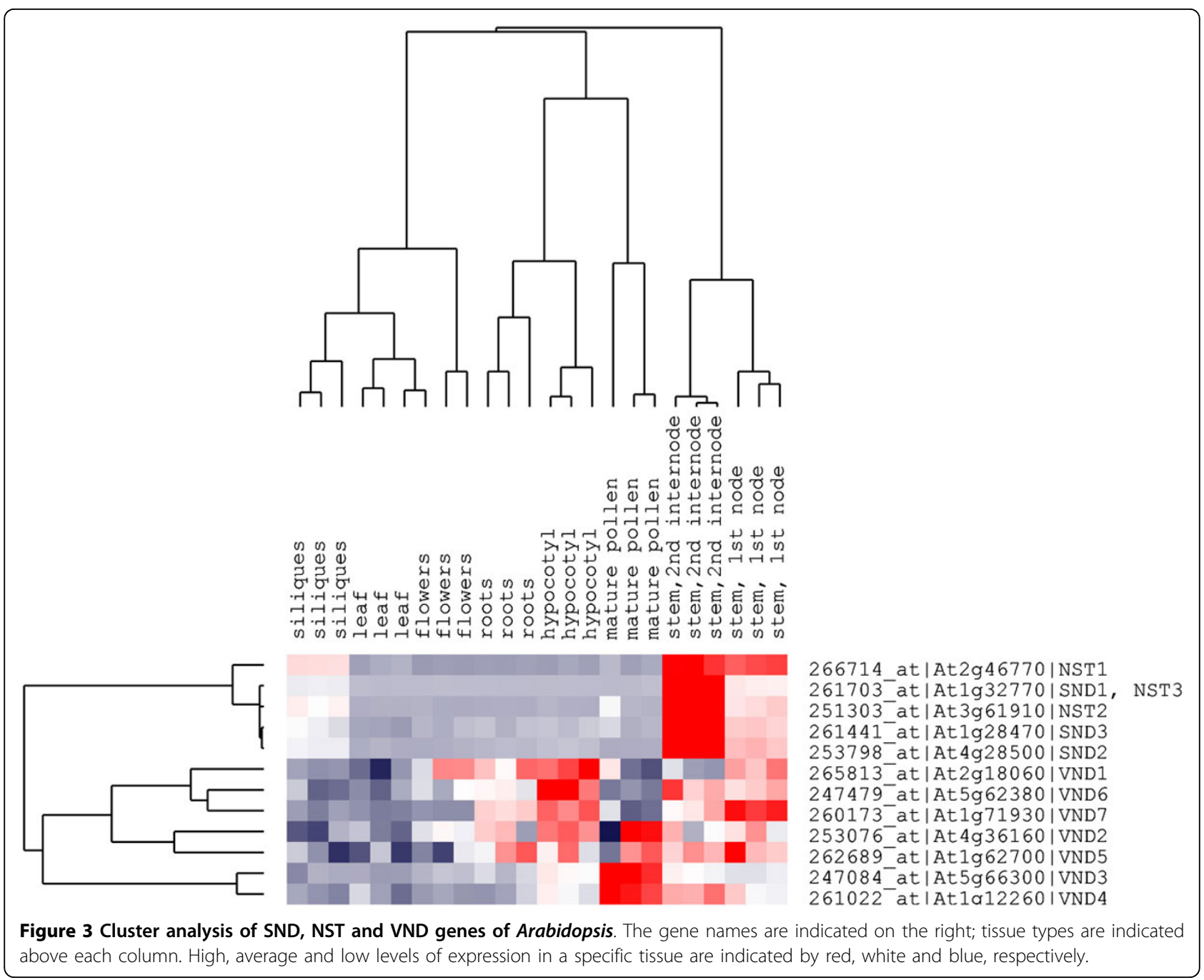

rice indica var. Minghui 63, and the gene expression patterns analysed for rice SND, NST and VND orthologue genes that possibly control cell wall composition. Using an approach similar to that employed for Arabidopsis microarray analysis, four rice NST and SND orthologue genes showed similar expression pattern in the tissue specific profiles (Figure 4). These four genes were significantly highly expressed in stem tissues, especially in the stem_2 (rice stem during heading stage) and were relatively less expressed in the leaves, endosperm, plumule, panicle and callus. The rice VND orthologue genes exhibited much more diverse expression patterns in these rice tissues. For example, LOC_Os06g04090 showed preferential expression in stem_2, callus and radicle. LOC_Os10g38834 was highly expressed in panicle, callus and radicle. We also analysed the expression profiles of rice SND, NST and VND orthologue genes in the developmental transcriptomes of var. Zhenshan 97, another rice indica variety, which showed similar results (data not shown) to those of var. Minghui 63. Real-time
RT-PCR experiments were used to further validate the microarray data for those rice NAC genes. Six SND, NST and VND orthologue genes were selected to examine the relative expression intensity in seven rice tissues, including callus, root, penultimate leaf, flag leaf, spikelet, seed and stem. The real-time RT-PCR results (Figure 5) mostly matched the microarray results. The expression pattern analysis of rice SND, NST and VND orthologue genes suggests the existence of conservation of tissue specificity in transcription levels between rice and Arabidopsis, especially for SND and NST orthologue genes with preferential expression in developing stem tissues.

\section{Co-regulatory network analysis for secondary cell wall biosynthesis NAC transcriptional factors SND1, SND2 and SND3}

The Arabidopsis tissue experiment clustering results showed that the SND genes were closely clustered together and displayed a specific expression in second internodes of the stem. The co-expression of genes 


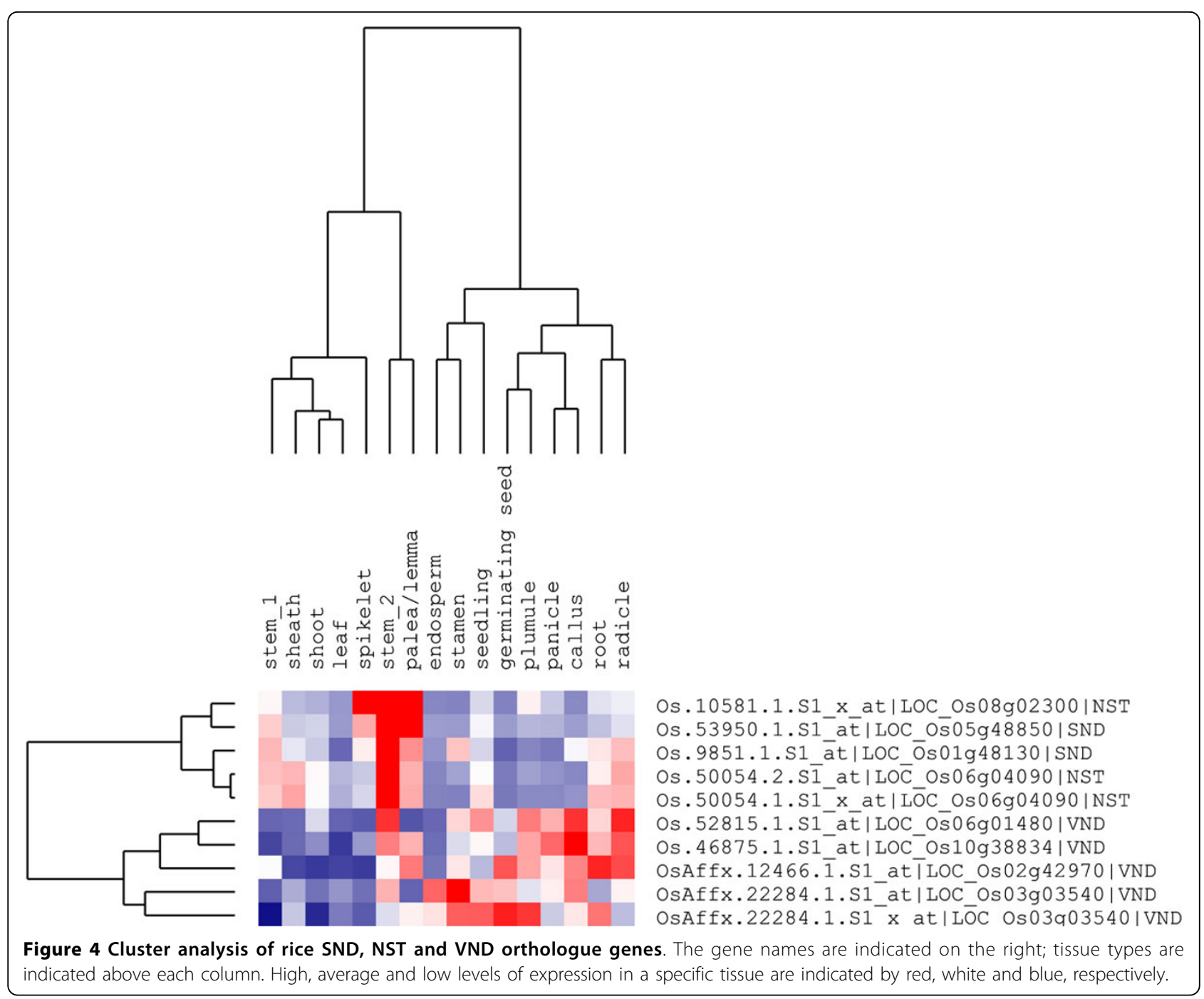

indicates that they may be co-regulated and involved in similar molecular regulatory pathways. We applied ATTED-II http://atted.jp/ and text mining results to predict the possible network where these three NAC transcriptional factors are involved in secondary cell wall biosynthesis. ATTED-II was built based on 1388 GeneChip data in Arabidopsis. The conditions were very diverse, including multiple tissue types, abiotic and biotic stress, hormone treatments and gene mutants [36]. Using Pathway Studio, we built a co-expression gene network for the three SND genes. Each SND gene was co-expressed with multiple other genes (Figure 6). The three SND genes coexpressed with some transcription factor genes, especially the MYB genes, e.g. MYB46, MYB52, MYB54, MYB58, MYB63, MYB69, MYB85 and MYB103. These MYB transcriptional factors are important in modulating cell wall biosynthesis [22]. For example, MYB46 is a direct target of SND1 and a key player in the transcriptional network involved in the regulation of secondary wall biosynthesis in Arabidopsis [37]. MYB58 was reported to directly activate the expression of lignin biosynthetic genes and a secondary wall-associated laccase (LAC4) gene. MYB58 and MYB63 are transcriptional activators of the lignin biosynthetic pathway during secondary cell wall formation in Arabidopsis and their expression was regulated by SND1 [38]. The Class II KNOX gene KNAT7 was also coexpressed with SND genes. KNAT7 was shown to negatively regulate secondary wall formation in Arabidopsis and is functionally conserved in Populus [39]. Some other transcription factors were also involved in this network, e.g. zinc finger, ERF38 and WRKY.

Furthermore, some secondary cell wall metabolismrelated genes were co-expressed with SND genes, such as LAC genes (LAC2, LAC5, LAC10, LAC12 and LAC17), IRX (IRREGULAR XYLEM) genes (IRX1, IRX3, IRX6, IRX9, IRX12 and IRX14), CESA4 (CELLULOSE SYNTHASE A4) and pectinase related protein. The knockout mutant of $L A C 2$ had been reported to reduce root elongation under 


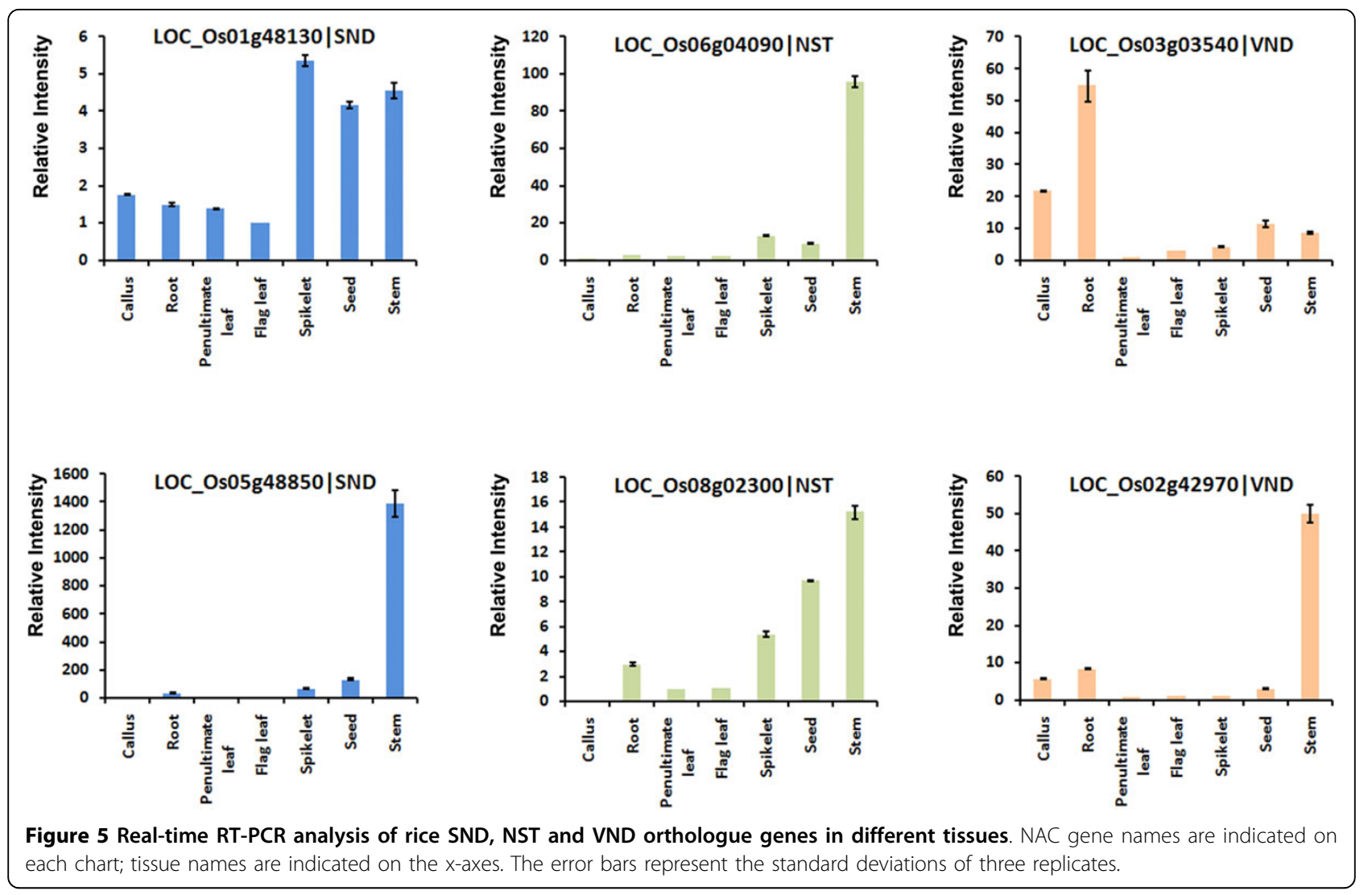

PEG-induced dehydration [40] and LAC17 mutants appear to have a reduced deposition of G lignin units [41]. IRX1 encodes a member of the cellulose synthase family [42-44], IRX3 encodes a xylem-specific cellulose synthase [45], IRX6 encodes a member of the COBRA family (similar to phytochelatin synthetase) [46], IRX9 encodes a putative family 43 glycosyl transferase [47,48], IRX12 (also known as LAC4) appears to have laccase activity [41], IRX14 encodes a putative family 43 glycosyl transferase [48,49], CESA4 encodes a cellulose synthase [46,50], and all these genes are involved in secondary cell wall biosynthesis.

Interestingly, several RIC (ROP-INTERACTIVE CRIB MOTIF-CONTAINING PROTEIN) genes were also coregulated with SND genes, e.g. RIC2 (involved in pollen tube growth and function [51]) and RIC4 (interacts with ROP2 during pavement cell morphogenesis and with ROP1 to promote apical F-actin assembly [52]). In addition, the co-regulatory network analysis also revealed that many novel genes were co-expressed with SNDs.

There were a total of 134 genes involved in this network (Supplemental Table 2 in additional file 2). GO analysis [30] was also performed for these 134 SND coregulated genes (Table 1) to decipher the possible biological pathways in which these genes were involved. Of the 134 genes queried, there were 131 genes with annotated GO items. We used 0.05 as the cut-off of FDR adjusted p-value. The most significantly enriched GO terms were 'secondary cell wall biogenesis process' (GO:0009834, FDR p-value $=2.40 \mathrm{E}-22$ ), 'cellular polysaccharide biosynthetic process' (GO:0033692, FDR $\mathrm{p}$-value $=4.30 \mathrm{E}-07)$, 'phenylpropanoid biosynthetic process' (GO:0009699, FDR p-value $=2.50 \mathrm{E}-06)$, 'transcription factor activity' (GO:0003700, FDR p-value = 3.00E-49) and 'laccase activity' (GO:0008471, FDR $\mathrm{p}$-value $=3.00 \mathrm{E}-09$ ). The GO terms related to other biological processes were also enriched, e.g. 'response to stress' (GO:0006950, FDR p-value $=5.60 \mathrm{E}-03$ ), 'oxidoreductase activity' (GO:0016491, FDR p-value $=4.00 \mathrm{E}-04)$, 'transition metal ion binding' (GO:0046914, FDR p-value = 3.50E-05) and transporter activity. Also, most genes were localized in nuclear and membrane parts.

Co-regulatory network analysis of SNDs and GO enrichment analysis indicated that most co-expressed genes were involved in secondary cell wall biogenesis, while we also found that some oxidoreductase activity and phenylpropanoid biosynthesis pathway genes were co-expressed with SND genes, e.g. peroxidase 64, NADPH oxidase and FLS2. Some processes such as carbohydrate synthesis and transport were coordinately regulated toward cell wall biosynthesis. There may be cross-talk between secondary wall biosynthesis and other biological processes. 


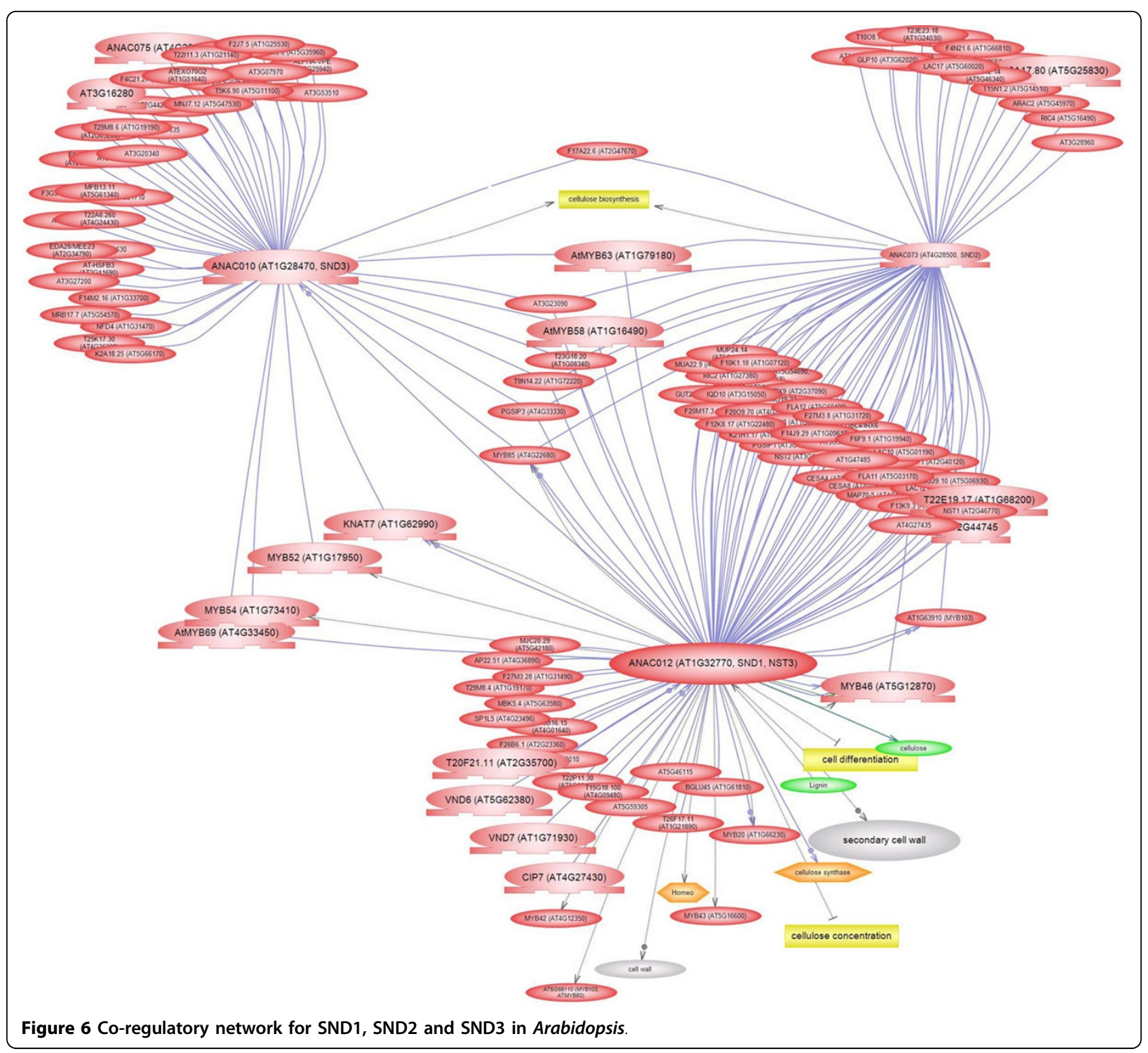

\section{Conclusions}

Combining the bioinformatics data mining and bench validation approach, we analysed the NST, VND and SND genes across plant species. The comparative genomic analysis revealed that the group VND of the NAC gene family evolutionarily first appeared in S. moellendorffii. The Arabidopsis and rice gene expression analysis showed the specific patterns of these NAC genes and the conservation of SNDs and NSTs in Arabidopsis and rice, and they were preferentially expressed in stems. The gene network analysis of SND genes in Arabidopsis showed that three SND genes (SND1, SND2 and SND3) co-expressed with multiple transcription factor genes, especially MYB genes and KNAT7, which are important in modulating cell wall biosynthesis. Additionally, the co-regulatory network analysis revealed many novel genes and pathways that could potentially be involved in cell wall biosynthesis and regulation. Nevertheless, there may be cross-talk between secondary wall biosynthesis and other biological process, such as stress response.

In summary, these results provided new insight into the evolution and the gene regulatory network of a subgroup of the NAC gene family controlling cell wall composition from the perspective of bioinformatics. These may help us to better understand the possible molecular mechanism underlying the regulation network of secondary cell wall biosynthesis and, therefore, improve plant biomass production. 


\section{Additional material}

Additional file 1: Supplemental Table 1. Primer list of rice SND, NST and VND orthologue genes for real-time RT-PCR (in doc format).

Additional file 2: Supplemental Table 2. The annotation of 134 genes involved in Arabidopsis SNDs-involved network (in xls format).

Additional file 3: Supplemental Table 3. The detailed information of 199 SND, NST and VND proteins studied across the 19 different species in this work (in xls format).

\section{Acknowledgements}

This work was supported by grants from the Ministry of Science and Technology of China (2012CB215300 and 31171276).

This article has been published as part of BMC Bioinformatics Volume 13 Supplement 15, 2012: Proceedings of the Ninth Annual MCBIOS Conference. Dealing with the Omics Data Deluge. The full contents of the supplement are available online at http://www.biomedcentral.com/bmcbioinformatics/ supplements/13/S15

\section{Author details}

${ }^{1}$ State Key Laboratory of Plant Physiology and Biochemistry, College of Biological Sciences, China Agricultural University, Beijing 100193, China. 2Department of Plant Pathology and Microbiology, Texas A\&M University, College Station, TX 77843, USA.

\section{Authors' contributions}

$W X, Z S$ and $J Y$ conceived and designed the study. QW performed the experiments. WX and DY analysed the data. WX, DY, ZS and RDS wrote the paper.

\section{Competing interests}

The authors declare that they have no competing interests.

Published: 11 September 2012

\section{References}

1. Yuan JS, Tiller KH, Al-Ahmad H, Stewart NR, Stewart CN Jr: Plants to power: bioenergy to fuel the future. Trends plant sci 2008, 13(8):421-429.

2. Li H, Cann AF, Liao JC: Biofuels: Biomolecular Engineering Fundamentals and Advances. Annu Rev Chem Biomol Eng 2010, 1:19-36.

3. Keegstra MPaK: Plant cell wall polymers as precursors for biofuels. Curr Opin Plant Biol 2010, 13:305-312.

4. Abramson MSO, Shani Z: Plant cell wall reconstruction toward improved lignocellulosic production and processability. Plant Sci 2010, 178:61-72.

5. Himmel ME, Ding SY, Johnson DK, Adney WS, Nimlos MR, Brady JW, Foust TD: Biomass recalcitrance: engineering plants and enzymes for biofuels production. Science 2007, 315(5813):804-807.

6. Ragauskas AJ, Williams CK, Davison BH, Britovsek G, Cairney J, Eckert CA, Frederick WJ Jr, Hallett JP, Leak DJ, Liotta CL, et al: The path forward for biofuels and biomaterials. Science 2006, 311(5760):484-489.

7. Simmons BA, Loque D, Ralph J: Advances in modifying lignin for enhanced biofuel production. Curr Opin Plant Biol 2010, 13(3):313-320.

8. Souer E, van Houwelingen A, Kloos D, Mol J, Koes R: The no apical meristem gene of Petunia is required for pattern formation in embryos and flowers and is expressed at meristem and primordia boundaries. Cell 1996, 85(2):159-170.

9. Delessert C, Kazan K, Wilson IW, Van Der Straeten D, Manners J, Dennis ES, Dolferus R: The transcription factor ATAF2 represses the expression of pathogenesis-related genes in Arabidopsis. Plant J 2005, 43(5):745-757.

10. Lu PL, Chen NZ, An R, Su Z, Qi BS, Ren F, Chen J, Wang XC: A novel drought-inducible gene, ATAF1, encodes a NAC family protein that negatively regulates the expression of stress-responsive genes in Arabidopsis. Plant mol biol 2007, 63(2):289-305.

11. Aida M, Ishida T, Fukaki H, Fujisawa H, Tasaka M: Genes involved in organ separation in Arabidopsis: an analysis of the cup-shaped cotyledon mutant. The Plant cell 1997, 9(6):841-857.
12. Yamaguchi-Shinozaki K, Koizumi M, Urao S, Shinozaki K: Molecular Cloning and Characterization of 9 cDNAs for Genes That Are Responsive to Desiccation in Arabidopsis thaliana: SequenceAnalysis of One cDNA Clone That Encodes a Putative Transmembrane Channel Protein. Plant Cell Physiol 1992, 33(3):217-224.

13. Fujita M, Fujita Y, Maruyama K, Seki M, Hiratsu K, Ohme-Takagi M, Tran LS, Yamaguchi-Shinozaki K, Shinozaki K: A dehydration-induced NAC protein $\mathrm{RD} 26$, is involved in a novel ABA-dependent stress-signaling pathway. Plant J 2004, 39(6):863-876.

14. Collinge $M$, Boller $T$ : Differential induction of two potato genes, Stprx2 and StNAC, in response to infection by Phytophthora infestans and to wounding. Plant mol biol 2001, 46(5):521-529.

15. Nakashima K, Tran LS, Van Nguyen D, Fujita M, Maruyama K, Todaka D, Ito Y, Hayashi N, Shinozaki K, Yamaguchi-Shinozaki K: Functional analysis of a NAC-type transcription factor OsNAC6 involved in abiotic and biotic stress-responsive gene expression in rice. Plant J 2007, 51(4):617-630.

16. Hu H, You J, Fang Y, Zhu X, Qi Z, Xiong L: Characterization of transcription factor gene SNAC2 conferring cold and salt tolerance in rice. Plant mol biol 2008, 67(1-2):169-181.

17. Hu R, Qi G, Kong Y, Kong D, Gao Q, Zhou G: Comprehensive analysis of NAC domain transcription factor gene family in Populus trichocarpa. BMC plant biol 2010, 10:145.

18. Zhong R, Richardson EA, Ye ZH: Two NAC domain transcription factors, SND1 and NST1, function redundantly in regulation of secondary wall synthesis in fibers of Arabidopsis. Planta 2007, 225(6):1603-1611.

19. Mitsuda N, Iwase A, Yamamoto H, Yoshida M, Seki M, Shinozaki K, OhmeTakagi M: NAC transcription factors, NST1 and NST3, are key regulators of the formation of secondary walls in woody tissues of Arabidopsis. The Plant cell 2007, 19(1):270-280.

20. Mitsuda N, Ohme-Takagi M: NAC transcription factors NST1 and NST3 regulate pod shattering in a partially redundant manner by promoting secondary wall formation after the establishment of tissue identity. Plant J 2008, 56(5):768-778.

21. Mitsuda N, Seki M, Shinozaki K, Ohme-Takagi M: The NAC transcription factors NST1 and NST2 of Arabidopsis regulate secondary wall thickenings and are required for anther dehiscence. The Plant cell 2005, 17(11):2993-3006.

22. Zhong $\mathrm{R}$, Lee $\mathrm{C}$, Zhou J, McCarthy $\mathrm{RL}$, Ye ZH: A battery of transcription factors involved in the regulation of secondary cell wall biosynthesis in Arabidopsis. The Plant cell 2008, 20(10):2763-2782.

23. Iwase A, Hideno A, Watanabe K, Mitsuda N, Ohme-Takagi M: A chimeric NST repressor has the potential to improve glucose productivity from plant cell walls. J biotechnol 2009, 142(3-4):279-284.

24. McCarthy RL, Zhong R, Ye ZH: MYB83 is a direct target of SND1 and acts redundantly with MYB46 in the regulation of secondary cell wall biosynthesis in Arabidopsis. Plant cell physiol 2009, 50(11):1950-1964.

25. Zhong R, Lee C, Ye ZH: Global Analysis of Direct Targets of Secondary Wall NAC Master Switches in Arabidopsis. Mol plant 2010, 3(6):1087-1103.

26. Zhao Q, Gallego-Giraldo L, Wang H, Zeng Y, Ding SY, Chen F, Dixon RA: An NAC transcription factor orchestrates multiple features of cell wall development in Medicago truncatula. Plant J 2010, 63(1):100-114.

27. Ohashi-Ito K, Oda Y, Fukuda H: Arabidopsis VASCULAR-RELATED NACDOMAIN6 directly regulates the genes that govern programmed cell death and secondary wall formation during xylem differentiation. The Plant cell 2010, 22(10):3461-3473.

28. Zhong $\mathrm{R}$, Lee $\mathrm{C}$, Ye ZH: Functional characterization of poplar woodassociated NAC domain transcription factors. Plant physiol 2010, 152(2):1044-1055.

29. Zhao C, Avci U, Grant EH, Haigler CH, Beers EP: XND1, a member of the NAC domain family in Arabidopsis thaliana, negatively regulates lignocellulose synthesis and programmed cell death in xylem. Plant J 2008, 53(3):425-436.

30. Zhou X, Su Z: EasyGO: Gene Ontology-based annotation and functional enrichment analysis tool for agronomical species. BMC genomics 2007, 8:246.

31. Ma S, Gong Q, Bohnert HJ: An Arabidopsis gene network based on the graphical Gaussian model. Genome res 2007, 17(11):1614-1625.

32. Raval M, Chattoo BB: Role of media constituents and proline in callus growth, somatic embryogenesis and regeneration of Oryza sativa cv Indica. Ind j exp biol 1993, 31(7):600-603. 
33. Chang S, Chen W, Yang J: Another formula for calculating the gene change rate in real-time RT-PCR. Mol biol rep 2009, 36(8):2165-2168.

34. Pylatuik JD, Fobert PR: Comparison of transcript profiling on Arabidopsis microarray platform technologies. Plant mol biol 2005, 58(5):609-624.

35. Wang L, Xie W, Chen Y, Tang W, Yang J, Ye R, Liu L, Lin Y, Xu C, Xiao J, et al: A dynamic gene expression atlas covering the entire life cycle of rice. Plant J 61(5):752-766

36. Obayashi T, Kinoshita K, Nakai K, Shibaoka M, Hayashi S, Saeki M, Shibata D, Saito K, Ohta H: ATTED-II: a database of co-expressed genes and cis elements for identifying co-regulated gene groups in Arabidopsis. Nucleic acids res 2007, , 35 Database issue: D863-869.

37. Zhong R, Richardson EA, Ye ZH: The MYB46 transcription factor is a direct target of SND1 and regulates secondary wall biosynthesis in Arabidopsis. The Plant cell 2007, 19(9):2776-2792.

38. Zhou J, Lee C, Zhong R, Ye ZH: MYB58 and MYB63 are transcriptional activators of the lignin biosynthetic pathway during secondary cell wall formation in Arabidopsis. The Plant cell 2009, 21(1):248-266.

39. Li E, Bhargava A, Qiang W, Friedmann MC, Forneris N, Savidge RA, Johnson LA, Mansfield SD, Ellis BE, Douglas CJ: The Class II KNOX gene KNAT7 negatively regulates secondary wall formation in Arabidopsis and is functionally conserved in Populus. New phytol 2012, 194(1):102-115.

40. Cai X, Davis EJ, Ballif J, Liang M, Bushman E, Haroldsen V, Torabinejad J, Wu Y: Mutant identification and characterization of the laccase gene family in Arabidopsis. J exp bot 2006, 57(11):2563-2569.

41. Berthet $S$, Demont-Caulet $N$, Pollet B, Bidzinski $P$, Cezard L, Le Bris $P$, Borrega N, Herve J, Blondet E, Balzergue S, et al: Disruption of LACCASE4 and 17 results in tissue-specific alterations to lignification of Arabidopsis thaliana stems. The Plant cell 2011, 23(3):1124-1137.

42. Chen Z, Hong X, Zhang H, Wang Y, Li X, Zhu JK, Gong Z: Disruption of the cellulose synthase gene, AtCesA8/IRX1, enhances drought and osmotic stress tolerance in Arabidopsis. Plant J 2005, 43(2):273-283.

43. Persson S, Caffall KH, Freshour G, Hilley MT, Bauer S, Poindexter P, Hahn MG, Mohnen D, Somerville C: The Arabidopsis irregular xylem8 mutant is deficient in glucuronoxylan and homogalacturonan, which are essential for secondary cell wall integrity. The Plant cell 2007, 19(1):237-255

44. Turner SR, Somerville CR: Collapsed xylem phenotype of Arabidopsis identifies mutants deficient in cellulose deposition in the secondary cell wall. The Plant cell 1997, 9(5):689-701.

45. Bosca S, Barton CJ, Taylor NG, Ryden P, Neumetzler L, Pauly M, Roberts K, Seifert GJ: Interactions between MUR10/CesA7-dependent secondary cellulose biosynthesis and primary cell wall structure. Plant physio/ 2006 142(4):1353-1363.

46. Brown DM, Zeef LA, Ellis J, Goodacre R, Turner SR: Identification of novel genes in Arabidopsis involved in secondary cell wall formation using expression profiling and reverse genetics. The Plant cell 2005 17(8):2281-2295

47. Bauer S, Vasu P, Persson S, Mort AJ, Somerville CR: Development and application of a suite of polysaccharide-degrading enzymes for analyzing plant cell walls. Proc Nat Acad Sci USA 2006, 103(30):11417-11422

48. Brown DM, Goubet F, Wong WW, Goodacre R, Stephens E, Dupree P, Turner SR: Comparison of five xylan synthesis mutants reveals new insight into the mechanisms of xylan synthesis. Plant J 2007, 52(6):1154-1168

49. Lee C, Teng Q, Huang W, Zhong R, Ye ZH: The Arabidopsis family GT43 glycosyltransferases form two functionally nonredundant groups essential for the elongation of glucuronoxylan backbone. Plant physiol 2010, 153(2):526-541

50. Pear JR, Kawagoe Y, Schreckengost WE, Delmer DP, Stalker DM: Higher plants contain homologs of the bacterial celA genes encoding the catalytic subunit of cellulose synthase. Proc Nat Acad Sci USA 1996, 93(22):12637-12642.

51. Wu G, Gu Y, Li S, Yang Z: A genome-wide analysis of Arabidopsis Ropinteractive CRIB motif-containing proteins that act as Rop GTPase targets. The Plant cell 2001, 13(12):2841-2856.

52. Fu Y, Gu Y, Zheng Z, Wasteneys G, Yang Z: Arabidopsis interdigitating cell growth requires two antagonistic pathways with opposing action on cell morphogenesis. Cell 2005, 120(5):687-700.
doi:10.1186/1471-2105-13-S15-S10

Cite this article as: Yao et al:: Comparative genomic analysis of NAC transcriptional factors to dissect the regulatory mechanisms for cell wall biosynthesis. BMC Bioinformatics 2012 13(Suppl 15):S10.

\section{Submit your next manuscript to BioMed Central and take full advantage of:}

- Convenient online submission

- Thorough peer review

- No space constraints or color figure charges

- Immediate publication on acceptance

- Inclusion in PubMed, CAS, Scopus and Google Scholar

- Research which is freely available for redistribution
C Biomed Central 